\title{
Treating speciation processes as complex traits
}

In Box 4 of their Review ${ }^{1}$, Noor and Feder listed ten examples of genes involved in forming a reproductive barrier that could lead to speciation events.

The authors wrote: "...we could be surprised in the future as more barriers are mapped and prove to involve mechanisms such as meiotic drive or genomic conflict to a greater extent than is currently appreciated" (page 858). One argument that stems from this statement is that there are many pathways that can result in speciation events, of which only a subset is fully appreciated so far.

In an attempt to investigate the genetic basis of speciation, one can treat speciation events in a similar way to complex traits: as in other complex traits, speciation involves both genetic and environmental factors and it is hard to tell whether some factors have more weight than others.

However, unlike in the case of many complex traits, there might be a hierarchy among the factors involved in speciation events owing to the basic requirements of the process; that is, factors involved in the formation of reproductive barriers will be ranked higher than factors involved in mere adaptation to specific cellular processes. In order to create a reproductive barrier, disruption of gene flow is required, which can be most efficiently created by systems that are important enough to have the potential to undergo tight-and-fast co-evolutionary processes. Such attributes can be included when ranking the importance of mechanisms that lead to the formation of new species. It is possible to apply ranking to factors involved in the formation of reproductive barriers: first, factors that form a 'solid' barrier, such as those involved in sperm-ovum recognition, will not allow the formation of an interspecific hybrid; second, factors that allow the formation of a hybrid, but will affect its competence in life and its ability to reproduce; and third, factors that allow the formation of a hybrid and will not affect its ability to live, but will dramatically affect the hybrid's fitness in such a way that it will be selected against and rapidly disappear from the population.

Applying such a ranking approach means that genetic factors involved in speciation processes might not be evenly weighted. Moreover, there could be factors that carry more than one of the characteristics required for the speciation process, which increases their importance for speciation. Previously, two such mechanisms have been discussed, and they are mentioned by Noor and Feder as examples: chromosomal aberrations and sperm-ovum recognition proteins ${ }^{2,3}$. An additional mechanism that might have equal importance to these two mechanisms is the oxidative phosphorylation (OXPHOS) system, one subunit of which (cytochrome c) was mentioned by Noor and Feder. We would like to point out several unique characteristics of mitochondrial OXPHOS that support a possible major role for its involvement in speciation: first, the mitochondrial-OXPHOS-driven energy production and translation machinery are encoded by both the nuclear and mitochondrial genomes, which differ tenfold in the pace of their sequence evolution ${ }^{4}$. This calls for tight co-evolution of the subunits and was shown to underlie poor compatibility of mitochondrial and nuclear-DNA-encoded subunits from closely related species $^{5,6}$. Second, both nuclear- and mitochondrialDNA-encoded OXPHOS subunits are subjected to evolutionary adaptive processes (positive selection) $)^{7,8}$. Third, mutations in mitochondrial OXPHOS subunits reduce reproductive potential in vertebrates and invertebrates $^{9-11}$.

Taken together, regarding the speciation process as a complex trait, with some factors having a greater impact than others, might help to derive a model for the genetic basis of speciation, and assist in the discovery of novel speciation-associated mechanisms.

\section{Dan Mishmar and Moran Gershoni}

Department of Life Sciences, Ben-Gurion University of the Negev, Beer-Sheva, Israel 84105.

Correspondence to D.M. e-mail:dmishmar@bgu.ac.il

1. Noor, M. A. F. \& Feder, J. L. Speciation genetics: evolving approaches. Nature Rev. Genet. 7, 851-861 (2006).

2. Hey, J. Speciation and inversions: chimps and humans. BioEssays 25, 825-828 (2003).

3. Swanson, W. J. \& Vacquier, V. D. The rapid evolution of reproductive proteins. Nature Rev. Genet. 3, 137-144 (2002).

4. Lynch, M., Koskella, B. \& Schaack, S. Mutation pressure and the evolution of organelle genomic architecture. Science $\mathbf{3 1 1}$, 1727-1730 (2006). 
5. Barrientos, A., Kenyon, L. \& Moraes, C. T. Human xenomitochondrial cybrids. Cellular models of mitochondrial complex I deficiency. J. Biol. Chem. 273, 14210-14217 (1998).

6. Kenyon, L. \& Moraes, C. T. Expanding the functional human mitochondrial DNA database by the establishment of primate xenomitochondrial cybrids. Proc. Natl Acad. Sci. USA $\mathbf{9 4}$, 9131-9135 (1997).

7. Grossman, L. I., Wildman, D. E., Schmidt, T. R. \& Goodman, M. Accelerated evolution of the electron transport chain in anthropoid primates. Trends Genet. 20, 578-585 (2004).

8. Mishmar, D. et al. Adaptive selection of mitochondrial complex I subunits during primate radiation. Gene 378, 11-18 (2006).

9. McFarland, R. et al. Multiple neonatal deaths due to a homoplasmic mitochondrial DNA mutation. Nature Genet. 30, 145-146 (2002).

10. Willett, C. S. \& Burton, R. S. Evolution of interacting proteins in the mitochondrial electron transport system in a marine copepod. Mol. Biol. Evol. 21, 443-453 (2004).

11. Sackton, T. B., Haney, R. A. \& Rand, D. M. Cytonuclear coadaptation in Drosophila: disruption of cytochrome c oxidase activity in backcross genotypes. Evolution 57, 23152325 (2003). 